\title{
EBV-LMP1 is involved in vasculogenic mimicry formation via VEGFA/VEGFR1 signaling in nasopharyngeal carcinoma
}

\author{
SAN XU ${ }^{12^{*}}, \mathrm{JU} \mathrm{BAI}^{1,2^{*}}, \mathrm{ZHOU}_{\mathrm{ZHUAN}}{ }^{1,2^{*}}, \mathrm{BO} \mathrm{LI}^{3}, \mathrm{ZHIBAO}^{\mathrm{ZHANG}}{ }^{1,2}$ \\ XIA WU ${ }^{1,2}$, XIANGJIAN LUO ${ }^{1,2}$ and LIFANG YANG ${ }^{1,2}$ \\ ${ }^{1}$ Key Laboratory of Carcinogenesis and Cancer Invasion of the Ministry of Education, Xiangya Hospital, \\ Central South University; ${ }^{2}$ Cancer Research Institute, School of Basic Medical Science, Central South University; \\ ${ }^{3}$ Pathology Department, Xiangya Hospital, Central South University, Changsha, Hunan 410078, P.R. China
}

Received November 14, 2017; Accepted April 26, 2018

DOI: $10.3892 /$ or.2018.6414

\begin{abstract}
The Epstein-Barr virus latent membrane protein 1 (EBV-LMP1) is an oncoviral protein that plays an important role in oncogenic transformation in EBV-associated nasopharyngeal carcinoma (NPC). Our previous studies demonstrated that LMP1 increased VEGFA expression and upregulated angiogenesis in NPC. Vasculogenic mimicry (VM) is a mechanism by which tumor cells can obtain nutrients to survive, and VM has been observed in numerous types of tumors. However, the occurrence and significance of VM in NPC and the relationship between LMP1 and VM have not yet been evaluated. In the present study, we observed that it was almost impossible for LMP1-negative NPC cells to form tubular structures, whereas LMP1-positive NPC cells were able to form tubular structures. Moreover, VEGFA was found to be involved in VM formation in LMP1-positive NPC cells. Knockdown of LMP1 or VEGFR1 distinctly disrupted tubular structures, whereas inhibition of VEGFR2 did not affect the process, indicating that VEGFR1 not VEGFR2 signaling was involved in LMP1-mediated VM formation. Furthermore, the data of immunohistochemistry (IHC) and CD34/PAS double staining in a tumor tissue array showed that LMP1 was positively correlated with VEGFR1 and VM. Meanwhile, after analyzing the clinicopathological features, we found that VM formation was associated with a poor prognosis in NPC patients. These results suggest that VM formation is increased by EBV-LMP1 via VEGF/VEGFR1 signaling and provide additional information to clarify the role of EBV-LMP1 in NPC pathophysiology.
\end{abstract}

Correspondence to: Professor Lifang Yang, Cancer Research Institute, School of Basic Medical Science, Central South University, 110 Xiangya Road, Changsha, Hunan 410078, P.R. China

E-mail: yanglifang99@hotmail.com

${ }^{*}$ Contributed equally

Key words: LMP1, VEGFR1, VEGFR2, vasculogenic mimicry, nasopharyngeal carcinoma

\section{Introduction}

Angiogenesis represents a crucial event under both physiological and pathological conditions (1). In tumors, in addition to traditional angiogenesis, vasculogenic mimicry (VM) provides another mechanism by which tumor cells can obtain nutrients to survive (2). VM defines the ability of highly invasive tumor cells to form matrix-rich networks containing channels; these vascular channels lack an endothelial cell (EC) lining and have a basement membrane that stains positive with periodic acid-Schiff (PAS) reagent (3). It has been recognized that VM is not only involved in proliferation and metastatic potential but is also associated with a poor patient prognosis in numerous types of tumors, including melanoma, hepatoma, breast carcinoma, prostatic carcinoma, lung carcinoma, colorectal cancer and ovarian carcinoma $(4,5)$.

Since the introduction of VM in 1999 as a novel paradigm for tumor perfusion, many studies have contributed new insights into the underlying molecular pathways supporting VM. It has been established that vascular endothelial growth factor A (VEGFA) plays a crucial role in the formation of VM (6-8). VEGFA binds and activates two tyrosine kinase receptors, VEGF receptor 1 and 2 (VEGFR1 and VEGFR2). Some reports have shown that VEGFR2 combines with VEGFA in an autocrine or paracrine manner and possesses many signaling capacities in the formation of VM (9-11). In contrast with these findings, other studies have shown that VEGFR1 kinase influences VM formation independent of VEGFR2 (12,13). These studies suggest that there are different molecular mechanisms involved in different tumor cell types.

Nasopharyngeal carcinoma (NPC) is endemic in Southern China and Southeast Asia. Approximately $80 \%$ of NPC patients are infected with the Epstein-Barr virus (EBV); EBV encodes latent membrane protein 1 (LMP1) which is believed to play a key role in the pathogenesis of NPC (14). LMP1 activates various downstream oncogenic signaling pathways and induces various downstream pathological changes in the processes of cell proliferation, anti-apoptosis and metastasis (15). In addition, a clear correlation between LMP1 and angiogenesis has been observed in NPC, and this effect is attributed to VEGF secretion via a mechanism involving the induction of cyclooxygenase $2(\mathrm{COX}-2)$ and hypoxia 
inducible factor $1 \alpha$ (HIF-1 $\alpha)(16,17)$. Our previous studies also demonstrated that LMP1 increased VEGFA expression and upregulated angiogenesis in NPC $(18,19)$. These reports suggest the possibility that LMP1 is involved in VM formation in NPC.

Although VM has been observed in numerous types of tumors, whether VM occurs in NPC and the mechanisms involved in its generation have not been well defined. In this study, we tested the roles of LMP1 and VEGFA/VEGFR signaling in VM formation and investigated the correlation between the LMP1-mediated upregulation of VM and the prognosis of NPC patients.

\section{Materials and methods}

Materials. The materials and reagents used in this study were purchased from the following providers. The Periodic Acid-Schiff (PAS) kit was purchased from Sigma-Aldrich (Merck KGaA, Darmstadt, Germany); Matrigel was obtained from BD Biosciences (Franklin Lakes, NJ, USA). Primary anti-VEGFA (sc-152), anti-VEGFR1 (sc-316), anti-VEGFR2 (sc-505) and anti- $\beta$-actin (sc-8432) polyclonal antibodies; VEGFA small interfering RNA (siRNA); VEGFR1 siRNA; and VEGFR2 siRNA were purchased from Santa Cruz Biotechnology Inc. (Santa Cruz, CA, USA). Anti-LMP1 antibody was purchased from Dako/Agilent (M0897; Agilent Technologies, Inc., Santa Clara, CA, USA); anti-CD34 antibody was purchased from Abcam Corp. (cat. no. ab81289; Cambridge, UK); and the HistoMouse-SP Broad Spectrum DAB kit was purchased from Invitrogen-Zymed (Thermo Fisher Scientific, Inc., Waltham, MA, USA).

Cell culture and small interfering RNA (siRNA) transfection. HK1 is an LMP1-negative NPC cell line, and HK1-LMP1 is a stable LMP1-integrated cell line (20). Cells were grown in Gibco $^{\text {TM }}$ RPMI-1640 medium (Thermo Fisher Scientific, Inc., Waltham, MA, USA) supplemented with $10 \%$ fetal bovine serum (FBS). Cells at $60-70 \%$ confluence were transfected with siRNA using Invitrogen ${ }^{\mathrm{TM}}$ Lipofectamine $^{\mathrm{TM}} 2000$ (Thermo Fisher Scientific, Inc.) according to the manufacturer's instructions.

In vitro tube formation assay. A Matrigel tube formation assay was developed for testing tubular structure formation. Briefly, we transfected HK1-LMP1 cells with targeted siRNA (LMP1, VEGFA, VEGFR1, or VEGFR2) and control siRNA (CON). Cells were trypsinized after $24 \mathrm{~h}$ and centrifuged at $600 \mathrm{x} \mathrm{g}$ for $5 \mathrm{~min}$. Approximately $1 \times 10^{5}$ cells were seeded in each well of a 24-well plate with $200 \mu \mathrm{l}$ of embedded Matrigel. Next, the cells were incubated for $8 \mathrm{~h}$, and the extent of tubular structure formation was examined using an inverted microscope (CKX41SF; Olympus Corp., Tokyo, Japan).

Western blot analysis. Cells were harvested and lysed at $4^{\circ} \mathrm{C}$ for $15 \mathrm{~min}$ in lysis buffer, and the protein concentration was determined by the Bradford protein assay (Bio-Rad Laboratories, Hercules, CA, USA) according to the manufacturer's instructions. Proteins were then separated by SDS-PAGE (4-20\% Mini-PROTEAN ${ }^{\circledR}$ TGX $^{\mathrm{TM}}$ Precast Protein Gels (Bio-Rad Laboratories) and transferred to a nitro- cellulose membrane (GE Healthcare, Piscataway, NJ, USA). Membranes were blocked with TBS-T $(20 \mathrm{mM}$ Tris-HCl, $\mathrm{pH} 7.4,137 \mathrm{mM} \mathrm{NaCl}$ and $0.1 \%$ Tween-20) containing 5\% non-fat milk for $1 \mathrm{~h}$ at room temperature (RT). Then, the membranes were incubated with primary antibodies (LMP1, dilution 1:250; VEGFA, dilution 1:250; VEGFR1, dilution 1:500; VEGFR2, dilution 1:500), followed by incubation with horseradish peroxidase-conjugated secondary antibody (cat. nos. sc-2005 or sc-2004; Santa Cruz Biotechnology) for $1 \mathrm{~h}$ at RT and visualization with an enhanced chemiluminescence detection kit (Pierce ECL; Thermo Fisher Scientific, Inc., Pittsburgh, PA, USA). The relative protein levels were quantified using ImageJ software (National Institutes of Health, Bethesda, MD, USA).

Immunofluorescence analysis. The cells were fixed and permeabilized with cold methyl alcohol $\left(-20^{\circ} \mathrm{C}\right)$ for $10 \mathrm{~min}$, and then blocked in 5\% donkey serum in PBS for $1 \mathrm{~h}$ and incubated with the primary antibody in PBS containing $1 \%$ BSA at $4^{\circ} \mathrm{C}$ overnight. The cells were washed 3 times with PBS, and incubated for 30 min with fluorochrome-conjugated secondary antibody (cat. nos. A-11001 or A-21207; Thermo Fisher Scientific, Inc., Waltham, MA, USA) for $30 \mathrm{~min}$. For fluorescence analysis, cell samples were visualized on a laser scanning confocal microscopy with appropriate emission filters (LSM 510 META; Carl Zeiss, Oberkochen, Germany).

Immunohistochemical analysis and CD34/PAS double staining. The NPC tissue array was purchased from Pantomics (Richmond, CA, USA), and the NPC paraffin-embedded tumor tissue samples, clinical details, and follow-up data were obtained from the Pathology Department of Xiangya Hospital from 2009 to 2015. The institutional review board of the Xiangya Hospital Ethics Committee approved the use of human samples in this study. IHC staining was performed using a HistoMouse-SP Broad Spectrum DAB kit (Thermo Fisher Scientific, Inc., Waltham, MA, USA) according to standard protocols. The signal was detected using a diaminobenzidine solution. A semi-quantitative evaluation of the positivity of each protein by IHC was performed using a previously defined method (21). The percentage of positive cells was divided into five grades (percentage scores): $0, \leq 10 \%$; $1,11-25 \% ; 2,26-50 \% ; 3,51-75 \%$; and $4,>75 \%$. The intensity of staining was divided into four grades (intensity scores): 0 , no staining; 1 , light brown; 2 , brown; and 3 , dark brown. Staining positivity was determined by the following formula: Overall score $=$ percentage score $\mathrm{x}$ intensity score. The stained sections were independently examined by two of the authors (Z.Z. and B.L.). Scores of 3 to 12 were considered positive for LMP1 and VEGFR1 expression. For CD34/PAS double staining, after IHC staining for CD34 as described above, the sections were washed with running water for $6 \mathrm{~min}$, incubated with PAS for $15 \mathrm{~min}$, and counterstained with hematoxylin. To quantify the differences in the density of VM, we calculated the total number of VMs in five fields for each tumor dot or section.

Statistical analysis. Statistical analyses were performed using the Student's t-test. The Kaplan-Meier method was used to estimate progression-free survival, and the log-rank test 
A
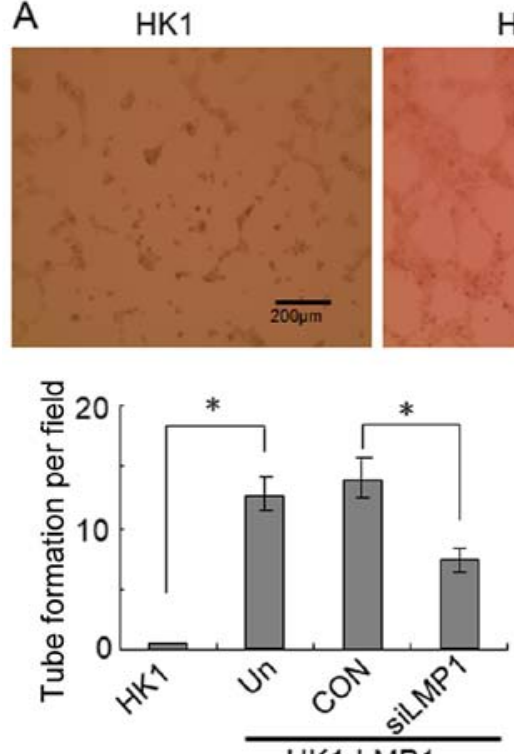

HK1-LMP1
HK1-LMP1

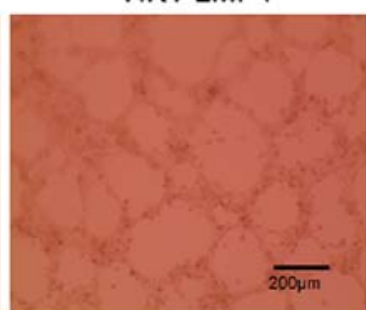

HK1-LMP1-CON

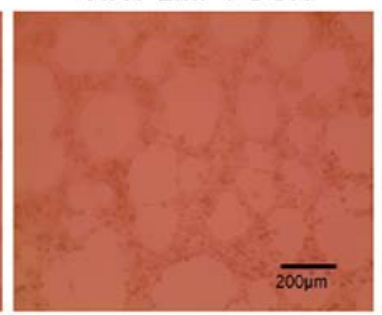

HK1-LMP1-siLMP1

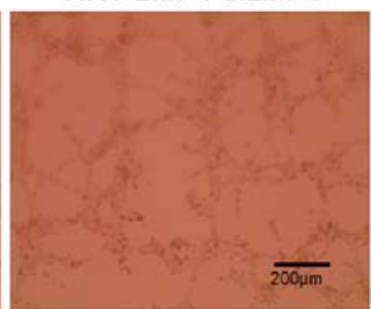

HK1-LMP1

HK1-LMP1
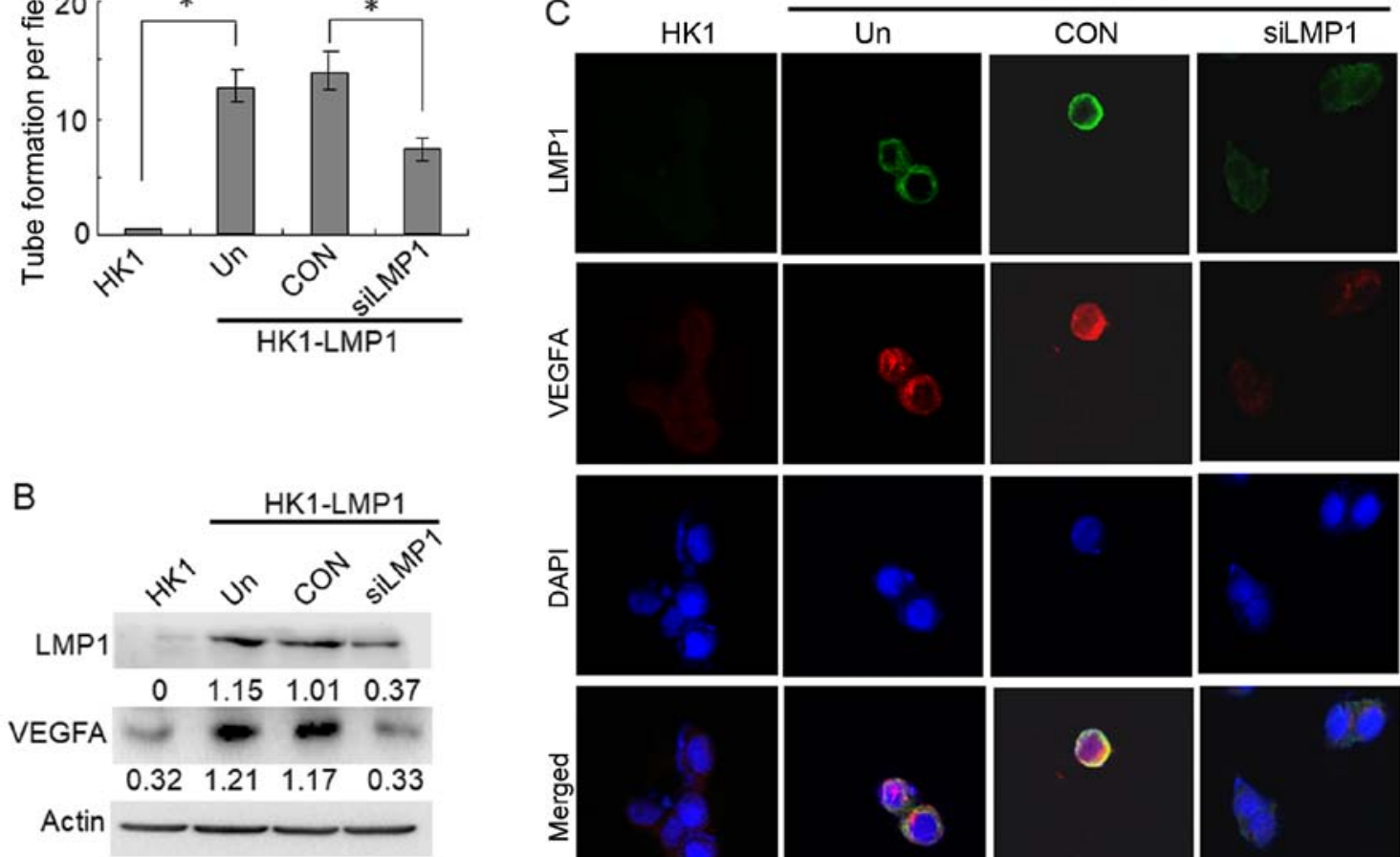
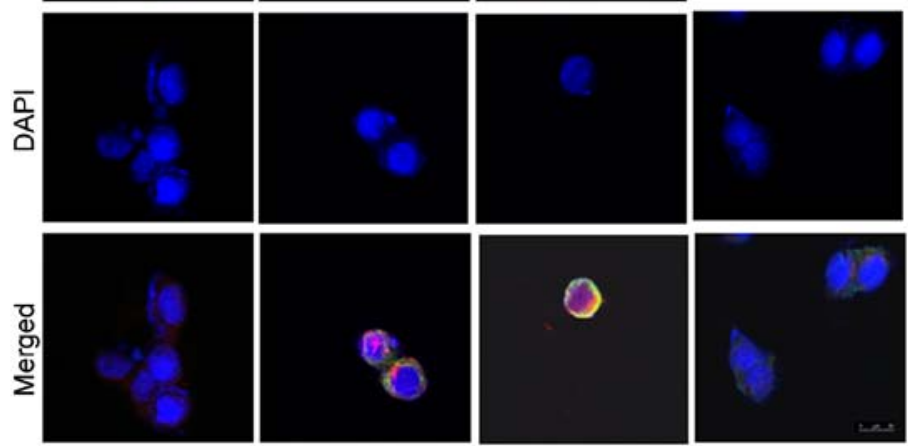

Figure 1. EBV-LMP1 is involved in the tubular structure formation in NPC cells and related to VEGFA expression. Cells were transfected with LMP1 siRNA for $24 \mathrm{~h}$. (A) Cells were trypsinized, collected, and cultured in a 24-well plate with $200 \mu 1$ of embedded Matrigel, and tubular structure formation was measured using light microscopy (x100) after $8 \mathrm{~h}$. The data show the average number of tubular structures in five random horizons. Data are expressed as the mean \pm SD.of three experiments, and an asterisk (") indicates a significant difference $(\mathrm{P}<0.05)$ compared with the control. (B) Western blot analysis of LMP1 and VEGFA protein expression; relative protein levels were quantified using ImageJ software. $\beta$-actin served as an internal control to confirm the equal loading of proteins. (C) Cells were fixed and stained with monoclonal antibodies to detect LMP1 (green) and VEGFA (red). The images were acquired using confocal microscopy. Representative images from 3 independent experiments are shown. EBV-LMP1, latent membrane protein 1; NPC, nasopharyngeal carcinoma; VEGFA, vascular endothelial growth factor A; Un, untreated; CON, control siRNA.

was used to evaluate differences between survival curves. A $\mathrm{P}$-value of $<0.05$ was considered statistically significant.

\section{Results}

EBV-LMPI is involved in the tubular structure formation in NPC cells and is related to VEGFA expression. Highly aggressive tumor cells form patterned networks of matrix-rich tubular structures when cultured on Matrigel (4). In this experiment, we used an in vitro tube formation assay to evaluate the tubular structure formation ability of different NPC tumor cells. The results showed that it was almost impossible for the LMP1-negative NPC cells, HK1, to form tubular structures in Matrigel, whereas the stable LMP1-integrated cells, HK1-LMP1, were able to form tubular structures. The tubular structure forming ability of the HK1-LMP1 cells decreased by approximately one-half after treatment with LMP1 siRNA compared with the control siRNA $(\mathrm{CON})$ group $(\mathrm{P}<0.05)$
(Fig. 1A). Thus, the data indicated that LMP1 might contribute to tumor VM formation in NPC.

Moreover, the western blotting results showed that VEGFA was highly expressed in the HK1-LMP1 cells compared to the level in the HK1 cells (Fig. 1B). Furthermore, when HK1-LMP1 cells were treated with LMP1 siRNA, LMP1 expression was reduced compared to that noted in the CON group, and this reduction was accompanied by decreased VEGFA expression. Meanwhile, the data of the immunofluorescence assay showed that, there was a higher VEGFA protein expression in HK1-LMP1 cells compared to that in the HK1 cells, and the co-localization of LMP1 and VEGFA was in the cytoplasm of NPC cells. Following inhibition of LMP1 expression by siRNA, both LMP1 and VEGFA expression was reduced compared to the CON group, and the co-localization of LMP1 and VEGF become very weak (Fig. 1C). These results indicated that EBV-LMP1 is involved in the tubular structure formation in NPC cells and is related to VEGFA expression. 

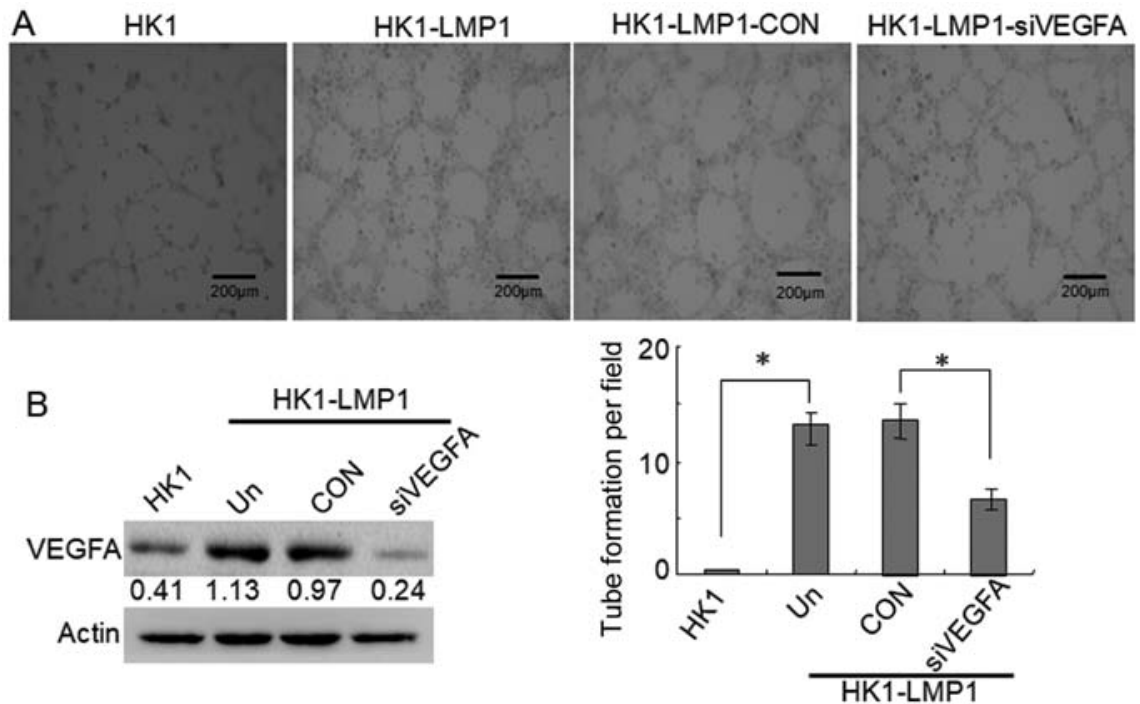

Figure 2. VEGFA contributes to LMP1-mediated tubular structure formation in NPC cells. Cells were transfected with VEGFA siRNA for 24 h. (A) Tubular structure formation was measured using light microscopy $(\mathrm{x} 100)$. The data show the average number of tubular structures in five random horizons. Data are expressed as the mean \pm SD of three experiments, and an asterisk (") indicates a significant difference $(\mathrm{P}<0.05)$ compared with the control. (B) Western blot analysis of VEGFA protein expression. Relative protein levels were quantified using ImageJ software. $\beta$-actin served as an internal control to confirm the equal loading of proteins. VEGFA, vascular endothelial growth factor A; LMP1, latent membrane protein 1; NPC, nasopharyngeal carcinoma; Un, untreated; CON, control siRNA.
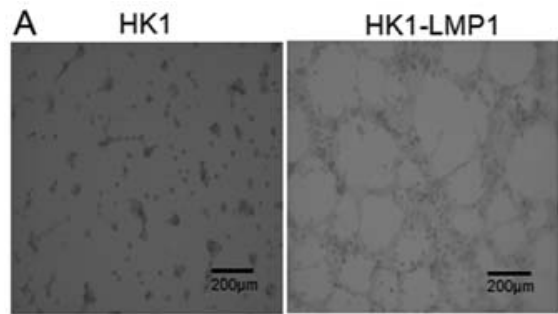

HK1-LMP1-CON HK1-LMP1-siVEGFR1

B

HK1-LMP1
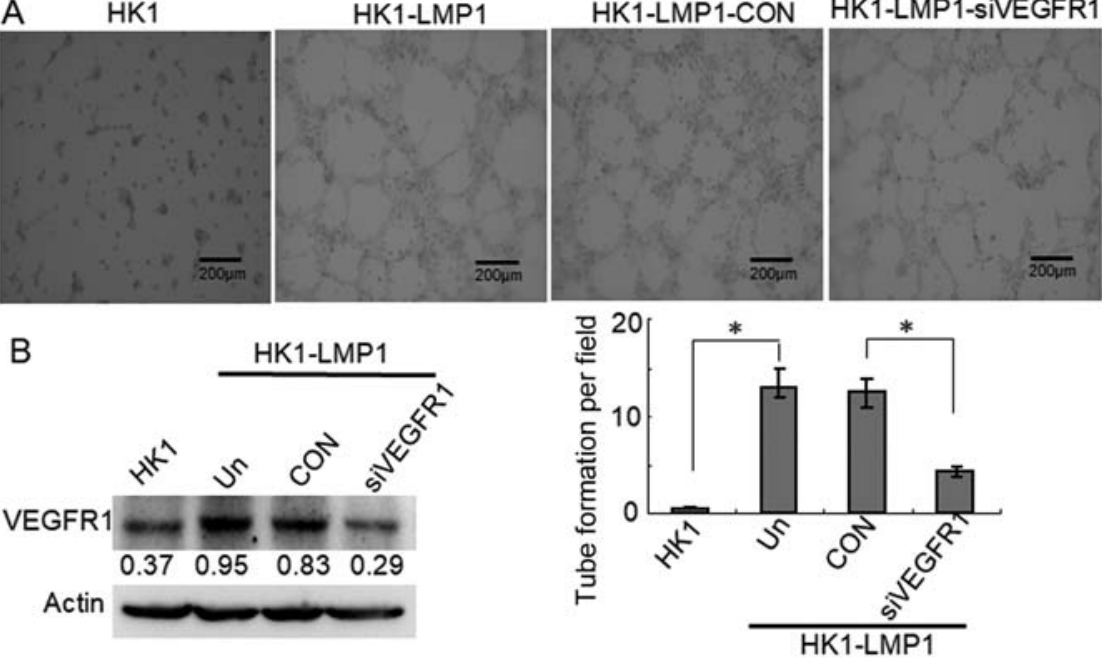

Figure 3. VEGFR1 is required for LMP1-mediated tubular structure formation in NPC cells. Cells were transfected with VEGFR1 siRNA for 24 h. (A) Tubular structure formation was measured using light microscopy (x100). The data show the average number of tubular structures in five random horizons. Data are expressed as the mean \pm SD of three experiments, and an asterisk ( $\left.{ }^{*}\right)$ indicates a significant difference $(\mathrm{P}<0.05)$ compared with the control. (B) Western blot analysis of VEGFR1 protein expression. Relative protein levels were quantified using ImageJ software. $\beta$-actin served as an internal control to confirm the equal loading of proteins. VEGFR1, vascular endothelial growth factor receptor 1; LMP1, latent membrane protein 1; NPC, nasopharyngeal carcinoma; Un, untreated; CON, control siRNA.

Furthermore, we investigated the contribution of VEGFA to tubular structure formation in LMP1-positive NPC cells. The data showed that VEGFA siRNA also strongly inhibited the formation of tubular structures compared with the CON group in the in vitro tube formation assays $(\mathrm{P}<0.05)$ (Fig. 2A). Meanwhile, VEGFA was highly expressed in HK1-LMP1 cells compared to that noted in the HK1 cells, and VEGFA siRNA significantly reduced VEGFA expression compared with that noted in the CON group (Fig. 2B). These findings indicated that VEGFA is involved in VM formation in LMP1-positive NPC cells.
VEGFR1 but not VEGFR2 is required for LMP1-mediated tubular structure formation in NPC cells. VEGFA performs its biological function mainly through binding and activating its receptor VEGFRs, VEGFR1 and VEGFR2, which have been shown to be involved in VM formation in different tumor cells $(6,12)$. Thus, we determined which VEGFR is required for the tubular structure formation in human LMP1-positive NPC cells. The data showed that VEGFR1 siRNA also strongly inhibited the formation of tubular structures compared with the $\mathrm{CON}$ group in in vitro tube formation assays $(\mathrm{P}<0.05)$ (Fig. 3A). Furthermore, VEGFR1 was highly expressed in 

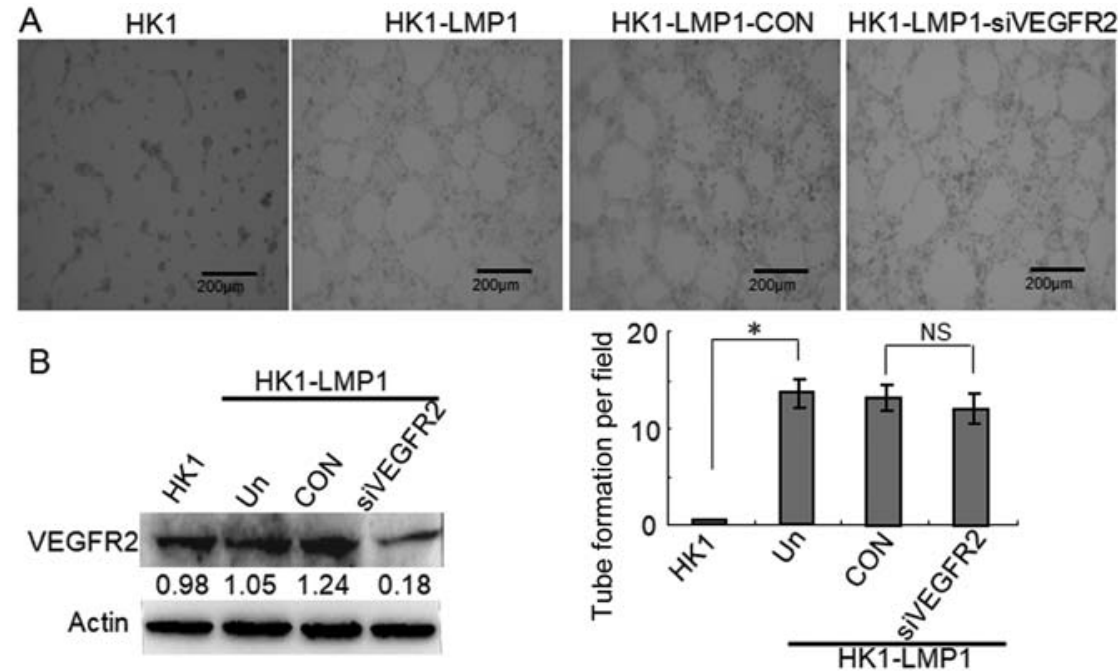

Figure 4. VEGFR-2 is not required for LMP1-mediated tubular structure formation in NPC cells. Cells were transfected with VEGFR2 siRNA for $24 \mathrm{~h}$. (A) Tubular structure formation was measured using light microscopy (x100). The data show the average number of tubular structures in five random horizons. Data are expressed as the mean $\pm \mathrm{SD}$ of three experiments, and an asterisk $\left(^{*}\right)$ indicates a significant difference $(\mathrm{P}<0.05)$ compared with the control. (B) Western blot analysis of VEGFR2 protein expression. Relative protein levels were quantified using ImageJ software. $\beta$-actin served as an internal control to confirm the equal loading of proteins. VEGFR2, vascular endothelial growth factor receptor 2; LMP1, latent membrane protein 1; NPC, nasopharyngeal carcinoma; Un, untreated; CON, control siRNA.

HK1-LMP1 cells compared to that observed in the HK1 cells. Moreover, VEGFR1 siRNA significantly reduced VEGFR1 expression compared with the CON group (Fig. 3B). These findings indicated that VEGRA/VEGFR1 signaling was involved in VM formation in LMP1-positive NPC cells. In contrast, HK1-LMP1 cells were transfected with VEGFR2 siRNA, which decreased the expression of VEGFR2. However, we did not observe any changes in the main geometrical features of the tubular structures that formed compared with the CON group (Fig. 4). These findings indicated that VEGFA/VEGFR2 signaling was not involved in the tubular structure formation in LMP1-positive NPC cells. According to the above western blotting results, VEGFA and VEGFR1 were significantly increased in LMP1-positive cells compared with LMP1-negative cells, whereas VEGFR2 did not change substantially. Therefore, we hypothesized that LMP1-VEGFA functions in the formation of VM through VEGFR1 rather than VEGFR2, which may be related to the proteins that are regulated by LMP1.

LMPI upregulates VEGFRI expression and VM formation in primary NPCs. The VM channels consist of a basement membrane with a lining of tumor cells on the external wall and do not contain endothelial cells (ECs) on the inner wall. Thus, PAS-positive and CD34-negative mature tumor vessels form a patterned network in the VM morphology (2). In further support of the preferential association of EBV-LMP1 and its downregulation of VEGFA/VEGFR1 signaling with VM in NPC, we examined the expression levels of LMP1 and VEGFR1 in a commercial NPC tissue array, and VM was detected in the tumor tissue array using CD34/PAS double staining. As shown in Fig. 5A, the VM channels were composed of NPC cells and were PAS-positive, CD34-negative (indicated by black arrows), while the endothelial-dependent vessels were positive for both CD34 and PAS (indicated by red arrows). These results showed that VM occurred in EBV-LMP1-positive
NPCs and that hardly any VM occurred in LMP1-negative NPCs. There was a positive correlation between LMP1 expression and VM formation according to the Pearson correlation coefficient results $(r=0.397, P=0.002)$. Meanwhile, the data showed a significant correlation between LMP1 and VEGFR1 expression (correlation coefficient $=0.481, \mathrm{P}=0.000$ ) (Fig. 5B). These results indicated that LMP1 expression was associated with VEGFR1 and VM formation in NPCs.

Presence of VM in tumor tissues is associated with a poor clinical outcome. The presence of VM in malignant tumors is associated with increased patient mortality (3). Thus, we determined whether the LMP1-mediated upregulation of VM is associated with the prognosis of NPC patients. We examined the expression levels of LMP1 and VM formation in tumor tissue samples from 40 NPC patients. These patients were successfully followed up (a median follow-up period of 5.61 years) (Table I). The positive staining rate of LMP1 in NPC tissues was $70 \%$ (28/40). The positive staining rate of VM in NPC tissues samples was $57.5 \%$ (23/40). There was a significant correlation between LMP1 expression and VM formation according to the Pearson correlation coefficient $(\mathrm{r}=0.372, \mathrm{P}=0.018)$. These results indicated that LMP1 expression is associated with VM formation in NPC tumor tissues, consistent with the results from the commercial NPC tissue array (Fig. 6A and B).

Using the clinical follow-up data, we retrospectively analyzed the prognostic significance of VM formation on the progression-free survival time (PFS) of 40 NPC patients, who received radiation therapy or concomitant chemoradiotherapy. As shown in Fig. 6C, the results of the Kaplan-Meier method analysis with log-rank test revealed a statistically significant difference in PFS between the 23 patients in the VM-positive group (78.3\% with expression of LMP1, median survival time of 37.12 months) and the 17 patients in the VM-negative group (58.9\% with expression of LMP1, median survival time 
A

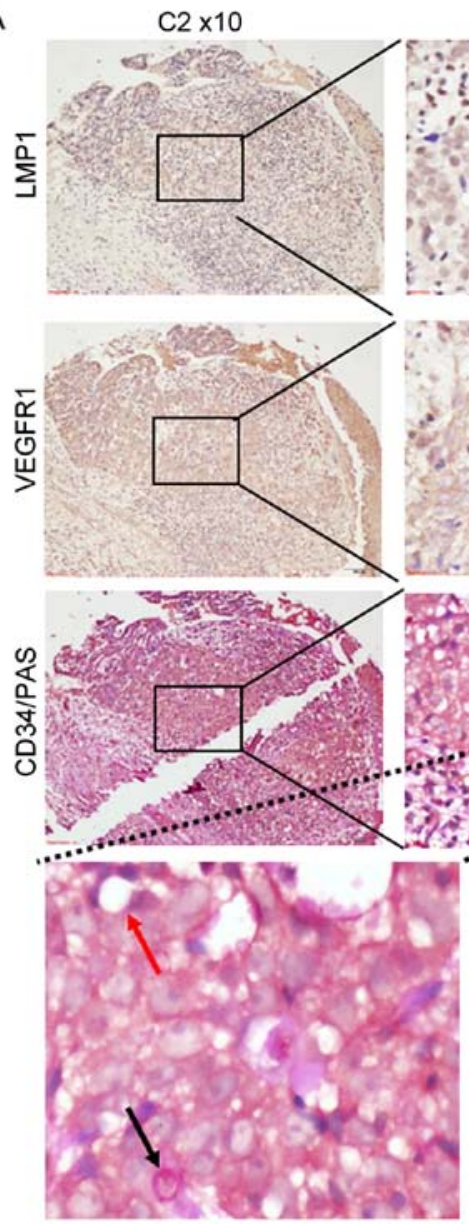

$\mathrm{C} 2 \times 20$
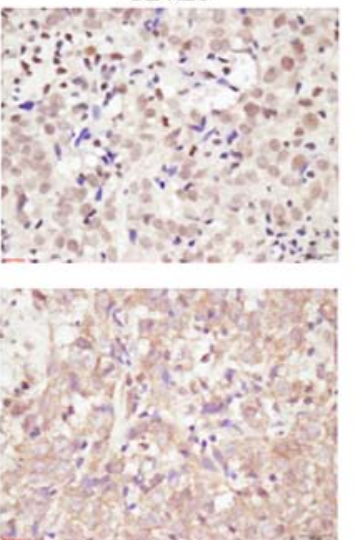

WA

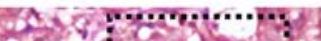

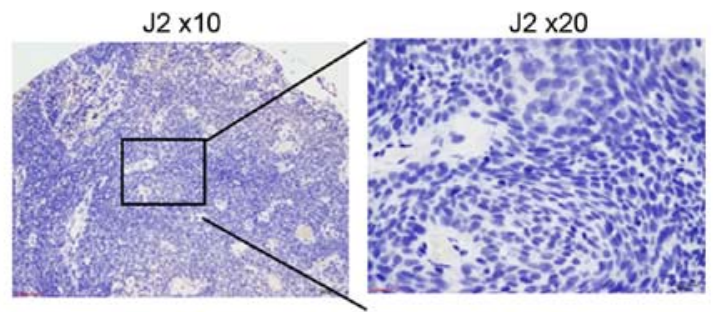
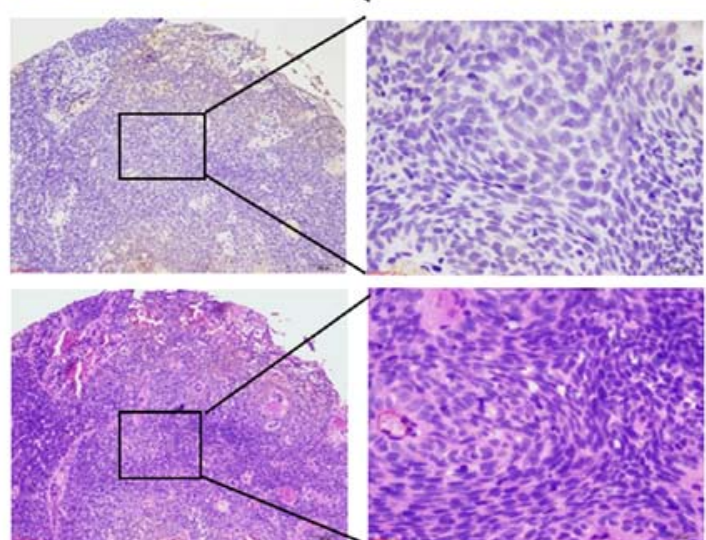

B

\begin{tabular}{llll}
\hline & & VEGFR1 & VM \\
\hline Spearman's Rho LMP1 & Correlation coefficient & $0.481^{* *}$ & $0.397^{*}$ \\
& Significant (2-tailed) & 0.000 & 0.002 \\
& $\mathrm{~N}$ & 61 & 61 \\
Spearman's Rho VEGFR1 & Correlation coefficient & $0.546^{* *}$ \\
& Significant (2-tailed) & 0.000 \\
& $\mathrm{~N}$ & 61 \\
\hline & *Correlation is significant at the 0.05 level (2-tailed) \\
** Correlation is significant at the 0.01 level(2-tailed).
\end{tabular}

Figure 5. LMP1 upregulates VEGFR1 expression and VM formation in an NPC tissue array. (A) Immunohistochemical analysis was performed to examine the levels of LMP1 and VEGFR1 expression, and CD34/PAS double staining was used to detect VM formation in an NPC tissue array (magnification, $\mathrm{x} 100$ and $\mathrm{x} 200$ ). The presence of PAS-positive and CD34-negative cells indicated VM channels, which were lined with tumor cells (black arrows). The endothelialdependent vessels were positive for both CD34 and PAS (red arrows). The tissue dot C2 exhibited high expression of LMP1 and VEGFR1 and was positive for VM, whereas tissue dot J2 exhibited low levels of LMP1 and VEGFR1 and was negative for VM. (B) Correlations between LMP1, VEGFR1, and VM formation in the NPC tissue array. Asterisks $\left({ }^{*}\right.$ or $\left.{ }^{* *}\right)$ indicates a significant (0.05, 2-tailed or 0.01, 2-tailed, respectively) correlation. LMP1, latent membrane protein 1; VEGFR1, vascular endothelial growth factor receptor 1; VM, vascular mimicry; NPC, nasopharyngeal carcinoma; PAS, periodic acid-Schiff.

A

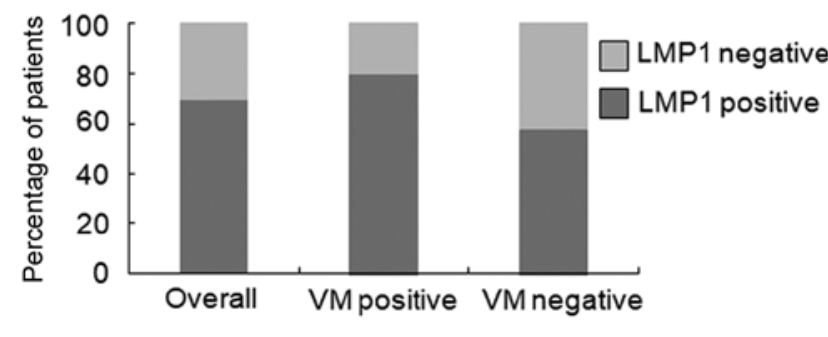

B

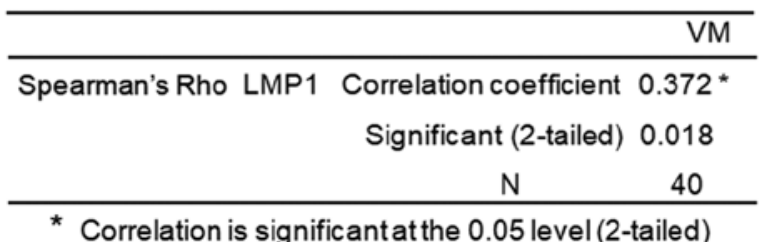

C

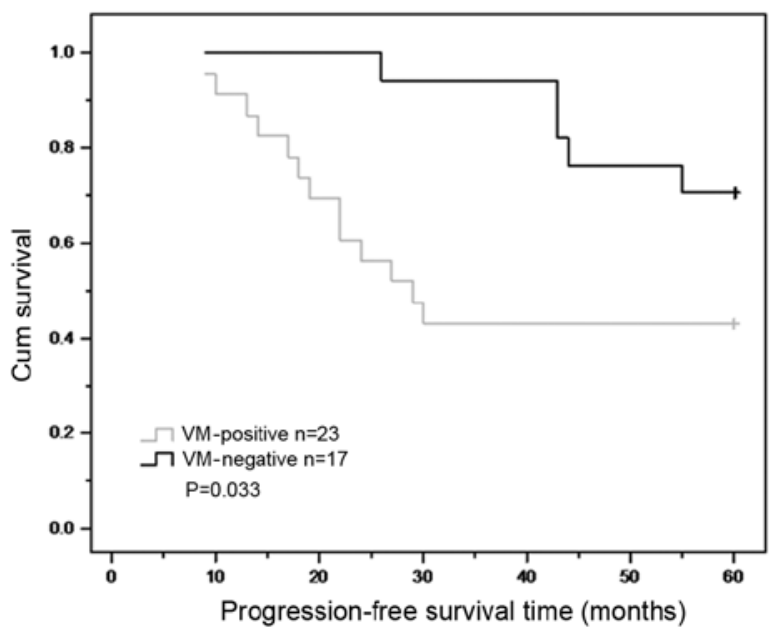

Figure 6. VM formation corresponds with poor progression-free survival of the NPC patients. Immunohistochemical analysis was used to examine the level of LMP1 expression, and CD34/PAS double staining was used to detect VM formation in tumor tissue samples from NPC patients. (A) The positive frequencies of LMP1 in the VM-positive and VM-negative groups are shown. (B) Correlation between VM formation and LMP1 expression in the NPC tumor tissue samples. (C) Progression-free survival rates of NPC patients with positive or negative VM formation were estimated with the Kaplan-Meier method with the log-rank test. VM, vascular mimicry; NPC, nasopharyngeal carcinoma; LMP1, latent membrane protein 1. 
Table I. Clinical characteristics of the NPC patients $(\mathrm{N}=40)$.

Characteristics

No. of patients (\%)

Sex
Male
Female
Age (years)
$>45$
$\leq 45$

Clinical stage

I

II-III

IV

Therapeutic modality

Radiation therapy

No treatment

$\mathrm{KSCC}$

$55 \%$ the activation of VEGFR1 and/or VEGFR2 results in cell proliferation, angiogenesis, and VM formation in tumors (6). The role of VEGFA/VEGFR signaling in regulating VM has been widely studied; however, it remains controversial. It is generally believed that VEGFA binds to VEGFR2 to trigger angiogenesis and VM formation in tumors $(9-13,22)$. In contrast with these findings, several reports identified the requirement of VEGFR1 for VM $(12,13)$. These discrepancies regarding the role of VEGFA/VEGFR signaling in modulating VM formation may reflect cell type- and/or cell line-specific differences. To gain a better understanding of the biological relevance of VEGFR subtypes on tubular network formation induced by EBV-LMP1, in our study, we used western blot assay, and the results showed that VEGFA and VEGFR1 were significantly increased in LMP1-positive cells compared with LMP1-negative cells, whereas VEGFR2 did not change substantially. We further used an in vitro tube formation assay and siRNA experiments, and the results suggested that VEGFR1 was the sole VEGFA receptor involved in signaling during VM formation in NPC. Therefore, we propose that LMP1-VEGFA functioned in the formation of VM through VEGFR1 rather than VEGFR2, which may be related to the proteins that are regulated by LMP1.

VM provides a sufficient blood supply for various malignant tumors to support their proliferation, invasion and metastasis (23). Some studies have shown that VM formation is significantly linked with a poor prognosis for patients with aggressive tumors, including melanoma, colorectal cancer, lung cancer, sarcomas and hepatic cancer (24-26). However, several studies have demonstrated that VM has no significant association with tumor prognosis, although a shorter survival time was observed in VM-positive patients $(27,28)$. Therefore, the influence of VM on cancer patient survival remains controversial. The results of our experiments not only indicated that VM was upregulated along with EBV-LMP1, but also suggested that VM formation was associated with a worse clinical outcome following therapy.

LMP1 has been shown to induce VEGFA production and increase angiogenesis in NPC tumors $(18,29)$. We previously reported that knockdown of LMP1 inhibited the expression and secretion of VEGFA through the JNK/c-Jun signaling pathway in NPC cells and enhanced the radiosensitivity of NPC cells by inhibiting HIF-1/VEGF activity (19). Collectively, EBV-LMP1 promotes angiogenesis in nasopharyngeal carcinoma, and also mediates the formation of VM, thereby providing nutrients for rapidly growing tumors. Further research of the molecular events underlying VM will offer new insights into the development of therapeutic strategies for EBV-LMP1-positive NPC.

\section{Acknowledgements}

Not applicable.

\section{Funding}

The present study was supported in part by the National Natural Science Foundation of China (nos. 81372182 and 81672761) and the Fundamental Research Funds for the Central

Universities of Central South University (no. 1053320170401). to the angiogenic growth factor family associated with tumor angiogenesis. The binding of VEGFA to its ligand and 


\section{Availability of data and materials}

The datasets used during the present study are available from the corresponding author upon reasonable request.

\section{Authors' contributions}

LY designed the research. SX, JB and ZZ performed the research and wrote the manuscript. All authors analysed the data and were involved in writing the manuscript. All authors read and approved the manuscript and agree to be accountable for all aspects of the research in ensuring that the accuracy or integrity of any part of the work are appropriately investigated and resolved.

\section{Ethics approval and consent to participate}

The present study was approved by the Institutional Review Board of the Xiangya Hospital Ethics Committee (Changsha, China).

\section{Consent for publication}

Not applicable.

\section{Competing interests}

The authors state that they have no competing interests.

\section{References}

1. Krishna Priya S, Nagare RP, Sneha VS, Sidhanth C, Bindhya S, Manasa P and Ganesan TS: Tumour angiogenesis-Origin of blood vessels. Int J Cancer 139: 729-735, 2016.

2. Zhang S, Zhang D and Sun B: Vasculogenic mimicry: Current status and future prospects. Cancer Lett 254: 157-164, 2007.

3. Maniotis AJ, Folberg R, Hess A, Seftor EA, Gardner LM, Pe'er J, Trent JM, Meltzer PS and Hendrix MJ: Vascular channel formation by human melanoma cells in vivo and in vitro: Vasculogenic mimicry. Am J Pathol 155: 739-752, 1999.

4. Qiao L, Liang N, Zhang J, Xie J, Liu F, Xu D, Yu X and Tian Y: Advanced research on vasculogenic mimicry in cancer. J Cell Mol Med 19: 315-326, 2015.

5. Williamson SC, Metcalf RL, Trapani F, Mohan S, Antonello J, Abbott B, Leong HS, Chester CP, Simms N, Polanski R, et al: Vasculogenic mimicry in small cell lung cancer. Nat Commun 7: 13322,2016

6. Kirschmann DA, Seftor EA, Hardy KM, Seftor RE and Hendrix MJ: Molecular pathways: vasculogenic mimicry in tumor cells: diagnostic and therapeutic implications. Clin Cancer Res 18: 2726-2732, 2012.

7. Schnegg CI, Yang MH, Ghosh SK and Hsu MY: Induction of vasculogenic mimicry overrides VEGF-A silencing and enriches stem-like cancer cells in melanoma. Cancer Res 75: 1682-1690, 2015.

8. Wang JY, Sun T, Zhao XL, Zhang SW, Zhang DF, Gu Q, Wang XH, Zhao N, Qie S and Sun BC: Functional significance of VEGF-a in human ovarian carcinoma: Role in vasculogenic mimicry. Cancer Biol Ther 7: 758-766, 2008.

9. Karroum A, Mirshahi P, Faussat AM, Therwath A, Mirshahi M and Hatmi M: Tubular network formation by adriamycinresistant MCF-7 breast cancer cells is closely linked to MMP-9 and VEGFR-2/VEGFR-3 over-expressions. Eur J Pharmacol 685: 1-7, 2012.

10. Yao X, Ping Y, Liu Y, Chen K, Yoshimura T, Liu M, Gong W, Chen C, Niu Q, Guo D, et al: Vascular endothelial growth factor receptor 2 (VEGFR-2) plays a key role in vasculogenic mimicry formation, neovascularization and tumor initiation by glioma stem-like cells. PLoS One 8: e57188, 2013.
11. Zhang S, Fu Z, Wei J, Guo J, Liu M and Du K: Peroxiredoxin 2 is involved in vasculogenic mimicry formation by targeting VEGFR2 activation in colorectal cancer. Med Oncol 32: 414, 2015.

12. Frank NY, Schatton T, Kim S, Zhan Q, Wilson BJ, Ma J, Saab KR, Osherov V, Widlund HR, Gasser M, et al: VEGFR-1 expressed by malignant melanoma-initiating cells is required for tumor growth. Cancer Res 71: 1474-1485, 2011.

13. Vartanian A, Stepanova E, Grigorieva I, Solomko E, Baryshnikov A and Lichinitser M: VEGFR1 and PKCa signaling control melanoma vasculogenic mimicry in a VEGFR2 kinase-independent manner. Melanoma Res 21: 91-98, 2011.

14. Young LS, Yap LF and Murray PG: Epstein-Barr virus: More than 50 years old and still providing surprises. Nat Rev Cancer 16: 789-802, 2016

15. Wang LW, Jiang S and Gewurz BE: Epstein-Barr virus LMP1-mediated oncogenicity. J Virol 91: 91, 2017.

16. Kondo S, Seo SY, Yoshizaki T, Wakisaka N, Furukawa M, Joab I, Jang KL and Pagano JS: EBV latent membrane protein 1 up-regulates hypoxia-inducible factor 1alpha through Siah1-mediated down-regulation of prolyl hydroxylases 1 and 3 in nasopharyngeal epithelial cells. Cancer Res 66: 9870-9877, 2006.

17. Murono S, Inoue H, Tanabe T, Joab I, Yoshizaki T, Furukawa M and Pagano JS: Induction of cyclooxygenase-2 by Epstein-Barr virus latent membrane protein 1 is involved in vascular endothelial growth factor production in nasopharyngeal carcinoma cells. Proc Natl Acad Sci USA 98: 6905-6910, 2001.

18. Wang Z, Luo F, Li L, Yang L, Hu D, Ma X, Lu Z, Sun L and Cao Y: STAT3 activation induced by Epstein-Barr virus latent membrane protein 1 causes vascular endothelial growth factor expression and cellular invasiveness via JAK3 And ERK signaling. Eur J Cancer 46: 2996-3006, 2010.

19. Yang L, Liu L, Xu Z, Liao W, Feng D, Dong X, Xu S, Xiao L, Lu J, Luo X, et al: EBV-LMP1 targeted DNAzyme enhances radiosensitivity by inhibiting tumor angiogenesis via the JNKs/HIF-1 pathway in nasopharyngeal carcinoma. Oncotarget 6: 5804-5817, 2015.

20. Xiao L, Hu ZY, Dong X, Tan Z, Li W, Tang M, Chen L, Yang L, Tao Y, Jiang Y, et al: Targeting Epstein-Barr virus oncoprotein LMP1-mediated glycolysis sensitizes nasopharyngeal carcinoma to radiation therapy. Oncogene 33: 4568-4578, 2014.

21. Hu J, Wang N and Wang YJ: XRCC3 and RAD51 expression are associated with clinical factors in breast cancer. PLoS One 8: e72104, 2013.

22. Holmes K, Roberts OL, Thomas AM and Cross MJ: Vascular endothelial growth factor receptor-2: Structure, function, intracellular signalling and therapeutic inhibition. Cell Signal 19: 2003-2012, 2007.

23. Hendrix MJ, Seftor EA, Seftor RE, Chao JT, Chien DS and Chu YW: Tumor cell vascular mimicry: Novel targeting opportunity in melanoma. Pharmacol Ther 159: 83-92, 2016.

24. Tan LY, Mintoff C, Johan MZ, Ebert BW, Fedele C, Zhang YF, Szeto P, Sheppard KE, McArthur GA, Foster-Smith E, et al: Desmoglein 2 promotes vasculogenic mimicry in melanoma and is associated with poor clinical outcome. Oncotarget 7: 46492-46508, 2016.

25. Bissanum R, Lirdprapamongkol K, Svasti J, Navakanitworakul R and Kanokwiroon K: The role of WT1 isoforms in vasculogenic mimicry and metastatic potential of human triple negative breast cancer cells. Biochem Biophys Res Commun 494: 256-262, 2017.

26. Sun J, Sun B, Sun R, Zhu D, Zhao X, Zhang Y, Dong X, Che N, Li J, Liu F, et al: HMGA2 promotes vasculogenic mimicry and tumor aggressiveness by upregulating Twist1 in gastric carcinoma. Sci Rep 7: 2229, 2017.

27. Massi D, Franchi A, Paglierani M, Ketabchi S, Borgognoni L, Reali UM and Santucci M: Vasculogenic mimicry has no prognostic significance in pT3 and pT4 cutaneous melanoma. Hum Pathol 35: 496-502, 2004

28. Shirakawa K, Wakasugi H, Heike Y, Watanabe I, Yamada S, Saito K and Konishi F: Vasculogenic mimicry and pseudocomedo formation in breast cancer. Int J Cancer 99: 821-828, 2002.

29. Yoshizaki T, Kondo S, Wakisaka N, Murono S, Endo K, Sugimoto H, Nakanishi S, Tsuji A and Ito M: Pathogenic role of Epstein-Barr virus latent membrane protein-1 in the development of nasopharyngeal carcinoma. Cancer Lett 337: 1-7, 2013. 